\title{
BMJ Open Ultrasound assessment for grading structural tendon changes in supraspinatus tendinopathy: an inter-rater reliability study
}

\author{
Kim Gordon Ingwersen, ${ }^{1,2}$ John Hjarbaek, ${ }^{3}$ Henrik Eshoej, ${ }^{1}$
} Camilla Marie Larsen, ${ }^{1,4}$ Jette Vobbe, ${ }^{5}$ Birgit Juul-Kristensen ${ }^{1,6}$

To cite: Ingwersen KG, Hjarbaek J, Eshoej $\mathrm{H}$, et al. Ultrasound assessment for grading structural tendon changes in supraspinatus tendinopathy: an inter-rater reliability study. BMJ Open 2016;6:e011746 doi:10.1136/bmjopen-2016011746

- Prepublication history and additional material is available. To view please visit the journal (http://dx.doi.org/ 10.1136/bmjopen-2016011746).

Received 7 March 2016 Revised 25 April 2016 Accepted 28 April 2016

CrossMark

For numbered affiliations see end of article.

Correspondence to Dr Kim Gordon Ingwersen; kim.riis@rsyd.dk

\section{ABSTRACT}

Aim: To evaluate the inter-rater reliability of measuring structural changes in the tendon of patients, clinically diagnosed with supraspinatus tendinopathy (cases) and healthy participants (controls), on ultrasound (US) images captured by standardised procedures.

Methods: A total of 40 participants (24 patients) were included for assessing inter-rater reliability of measurements of fibrillar disruption, neovascularity, as well as the number and total length of calcifications and tendon thickness. Linear weighted $\kappa$, intraclass correlation (ICC), SEM, limits of agreement (LOA) and minimal detectable change (MDC) were used to evaluate reliability.

Results: 'Moderate—almost perfect' $\kappa$ was found for grading fibrillar disruption, neovascularity and number of calcifications ( $\mathrm{k} 0.60-0.96$ ). For total length of calcifications and tendon thickness, ICC was 'excellent' (0.85-0.90), with SEM (Agreement) ranging from 0.63 to $2.94 \mathrm{~mm}$ and $\mathrm{MDC}_{\text {(group) }}$ ranging from 0.28 to $1.29 \mathrm{~mm}$. In general, SEM, LOA and MDC showed larger variation for calcifications than for tendon thickness.

Conclusions: Inter-rater reliability was moderate to almost perfect when a standardised procedure was applied for measuring structural changes on captured US images and movie sequences of relevance for patients with supraspinatus tendinopathy. Future studies should test intra-rater and inter-rater reliability of the method in vivo for use in clinical practice, in addition to validation against a gold standard, such as MRI.

Trial registration number: NCT01984203; Pre-results.

\section{INTRODUCTION}

Rotator cuff (RC) tendinopathy can be considered a continuum of pathology, and tailored rehabilitation according to the stage in this continuum is recommended. ${ }^{12}$ Anamnesis and special orthopaedic tests are often used when diagnosing RC tendinopathy,

\section{Strengths and limitations of this study}

- A standardised procedure for US capturing and measuring structural changes of the supraspinatus tendon is presented.

- A specific procedure for grading and interpreting tendinopathy-related changes is presented.

- Grading and measurement can be performed reliably.

- Performance of the method in vivo is warranted to validate the method in clinical practice.

but these tests often lack high specificity and sensitivity, making diagnosis uncertain, ${ }^{3}$ thus challenging precise and targeted treatment.

Grey-scale (GS) ultrasound (US) and Power Doppler (PD) visualisation of RC tendons may be helpful to detect signs of tendinopathy, such as hypoechoic areas, fibrillar disruption (FD), neovascularisation $(\mathrm{NV})$, calcifications (CAs) embedded in the tendon or oedema, and confirm the 'a priori' hypothesis of RC tendinopathy, provided satisfactory clinimetric properties of the US method. ${ }^{4} 5$

However, US is an operator-dependent technique and requires thorough training and experience in performance and assessment before precise diagnoses can be made, especially in relation to more subtle changes as often seen within tendinopathy. ${ }^{6}$ Poor to fair reliability has previously been found when comparing US diagnoses made by novel and experienced clinicians. ${ }^{7-9}$ Further, when grading subtle structural tendon changes, especially hypoechoic areas, only fair and therefore unsatisfactory reliability has been found, even among experienced

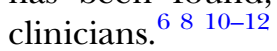

Standardised procedures for capturing and assessing US are known to increase reliability of US-based diagnoses. ${ }^{6}$ Previously, assessment 
of tendinopathy were found reliable, in patients with tendinopathy in the elbow, ankle or knee, when using standardised procedures for measuring GS and PD. ${ }^{411}$

For the shoulder, however, there is a lack of clinically relevant, standardised and reliable methods for assessing tendinopathy. Since US is highly influenced by clinician experience and technique, both standardised US procedures for image and movie capturing and standardised procedures for assessment of structural changes in relation to tendinopathy need to be defined.

Therefore, the aim of this study was to evaluate the inter-rater reliability of measuring and grading structural changes in the tendons of patients clinically diagnosed with supraspinatus tendinopathy (cases) and healthy participants (controls), on images and movies captured through standardised US procedures.

\section{MATERIALS AND METHODS}

\section{Study design}

The study followed the protocol for diagnostic procedures in reproducibility studies. ${ }^{13}$ This protocol includes a three-phase study design consisting of (1) training, (2) an overall agreement and (3) a study phase (the actual reliability study; figure 1 ).

The phases constitute a methodological model for optimising procedures, and aim at eliminating clinician subjectivity as much as possible. The aim of the training phase is to ensure that raters have sufficient competence and experience in performing the procedures. The overall agreement phase is an extended training phase and ensures that gross systematic bias between raters is minimised, and requires at least $80 \%$ agreement between raters before proceeding to phase 3 . The study phase is the final evaluation of reliability of the developed procedures. ${ }^{13}$

Inter-rater reliability (phase 3) between two raters (raters A and B) was tested on measuring and grading

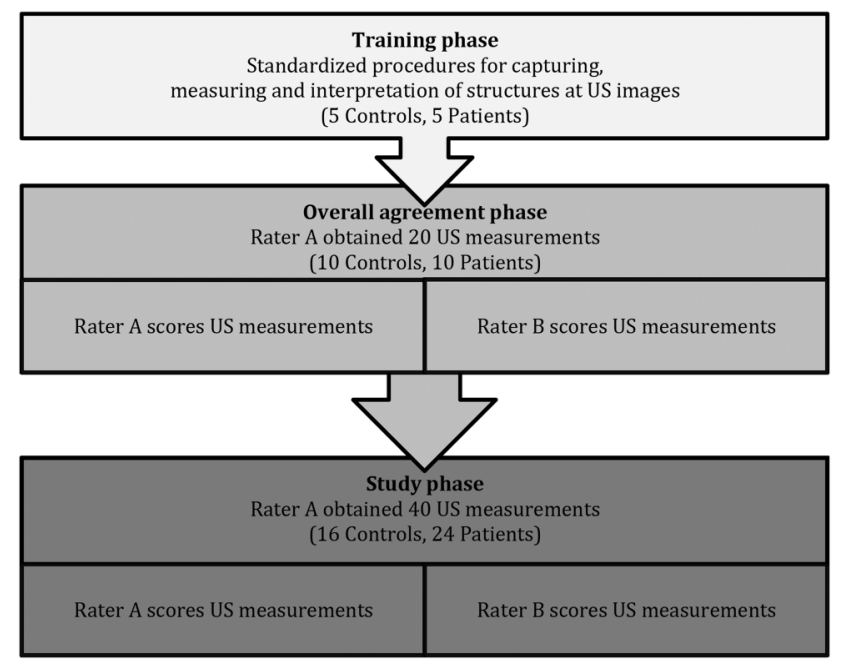

Figure 1 Flow chart of the training, overall agreement and study phase. US, ultrasound. structural changes relevant to tendinopathy on US-captured images and movies. Rater A (KGI; physiotherapist) had 1 year of clinical musculoskeletal US experience, and rater $\mathrm{B}(\mathrm{JH}$; radiologist $)$ had more than 15 years of clinical musculoskeletal US experience.

\section{US image capturing and measurement}

On the basis of the literature, ${ }^{4} 1011{ }^{14-17}$ consensus was made on definitions of relevant potential pathological structural changes related to tendinopathy, including (1) FD, (2) NV, (3) CA and (4) tendon thickness (TT). Hereafter, a standardised protocol for US capturing was developed, consisting of three static images (GS), three dynamic movie sequences (GS) and one Doppler movie sequence (table 1 ).

Second, on the basis of previous scales used to measure structural changes in tendinopathy at the elbow, ${ }^{4}{ }^{16}$ two ordinal grading scales for FD and $\mathrm{NV}$ were adjusted for use in the shoulder. ${ }^{19}$ The scales ranged from 0 to 4 (FD: $0=$ normal tendon; $4=$ partial rupture, corresponding to disruption of the fibres in the full thickness of the tendon; NV ( $0=$ normal, including no signal; $4=$ extreme, including Doppler activity in more than $50 \%$ of the region of interest, ROI; table 2; see online supplementary appendix).

CA was analysed as number of CAs and total length (in $\mathrm{mm}$ ), while TT was measured in $\mathrm{mm} .{ }^{18}$

Rater A performed capture of all US images and movie sequences with the participant seated, the shoulder internally rotated with the dorsal side of the hand placed on the sacrum, and the elbow flexed and directed laterally, to optimise visualisation of the supraspinatus tendon. ${ }^{20}$

A GE LOGIQ e B12 (GE Healthcare) with a 5.013.0 $\mathrm{MHz}$ linear transducer was used for image capturing. All US scannings were standardised and performed for GS imaging at $13.0 \mathrm{MHz}$ and $56 \%$ gain, while PD scanning was performed with a pulse repetition frequency of $0.41 \mathrm{kHz}$ and gain at $56 \%$. Manufacturer recommendations for musculoskeletal imaging of the shoulder were preset for the remaining parameters.

Captured images and movie sequences were stored with unique identifier labels on an external hard disk. Measurement of captured images and movie sequences was performed in 'OsiriX V.5.8.2 32-bit' (rater A) and RadiAnt DICOM viewer V.1.9.16 (32 bit; rater B).

In the overall agreement and study phase, raters were blinded to each other's results and the participant status (case/control), and images and movies were stored for at least 21 days before measurements to secure blinding of rater $\mathrm{A}$.

\section{Training and overall agreement phases}

In the training phase, raters A and B practised the US procedures for capturing, measuring and grading the captured images and movies on 10 participants (cases and controls). Overall agreement phase was performed 
Table 1 Description of US procedures for capturing image and movie sequences of FD, NV, CAs and TT

(1) FD:

FD was defined as a clear collagen fascicle discontinuity or irregularity of fibrils in an otherwise regular parallel structuring of fibres in the tendon.

A GS picture in the longitudinal axis of the supraspinatus was taken at the sight where FD was most apparent (FD picture). The FD static image was used for classifying the presence of FD. A GS PA dynamic movie sequence (PA movie) in the longitudinal plane of the supraspinatus tendon was captured by moving the transducer slowly in the PA direction. Further, a CC transversal GS dynamic movie sequence (CC movie) of the supraspinatus tendon was recorded by moving the transducer slowly in the $\mathrm{CC}$ direction. The static image and the movie sequence recordings were used as confirmation and assistance in assessing the grade of structural changes, and to secure identification of potential ambiguous GS features, such as anisotropy (erroneous signal caused when the transducer is angled obliquely to the tendon).

FD was classified in relation to tendon thickness as: $0=$ normal, $1=$ mild, $2=$ moderate, $3=$ severe, $4=$ partial rupture (table 2 ).

(2) NV:

NV was defined as a visualised PD signal with minimal artefactual noise. The supraspinatus tendon was evaluated for presence of NV by moving the transducer slowly in the PA direction, with the PD feature activated. In case NV was present, a $10 \mathrm{~s}$ dynamic movie sequence was recorded at the point with most NV signal (PD movie). When grading NV from the PD movie sequence, a static image of the location with the most visible NV was captured from the PD movie. A ROI $(5 \times 5 \mathrm{~mm})$ was placed around the NV and used for grading NV. In participants with no NV, a movie sequence was recorded at a random location in the tendon to verify absence of NV. NV was classified in relation to ROI as $0=$ normal, $1=$ mild, $2=$ moderate, $3=$ severe, $4=$ extreme (table 2 ).

(3) $C A$ :

CA was defined as distinct white borders, imbedded in the length of the tendon, often with 'shadows' underneath. The PA movie was used to identify the number of CA in the tendon and to measure the length of each CA in the longitudinal axis of the supraspinatus. The length was measured between the most medial and lateral aspects of the distinct white boarder (in $\mathrm{mm}$ ). The $\mathrm{CC}$ movie was used as confirmation and assistance in identifying CA. CA was counted and measured $(\mathrm{mm})$. To obtain the total length of CA, the individual CA lengths were added up to one total per participant.

(4) $T T$ :

TT was defined as the height, from the humeral head, at a point $20 \mathrm{~mm}$ from the supraspinatus tendon-snip (tendon insertion) in the longitudinal axis of the tendon, to the most superficial part of the tendon. In practice, an image was captured at a fixed point just laterally from the anterior-lateral corner of the acromion in the longitudinal plane of the supraspinatus. When measuring TT, a mark was placed $20 \mathrm{~mm}$ cranial from the supraspinatus tendon-snip (tendon insertion), on the edge of the cartilage of the humeral head. From that mark, the perpendicular thickness of the tendon was recorded. ${ }^{18}$ The TT picture was recorded bilaterally. TT was measured in $\mathrm{mm}$.

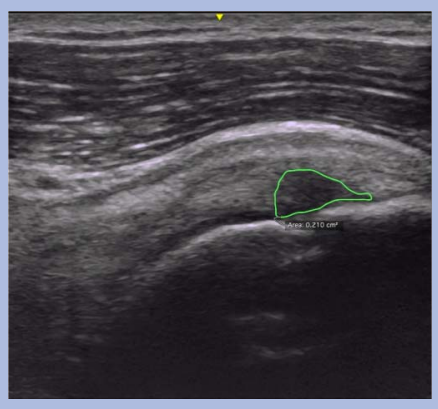

Grade 2 FD

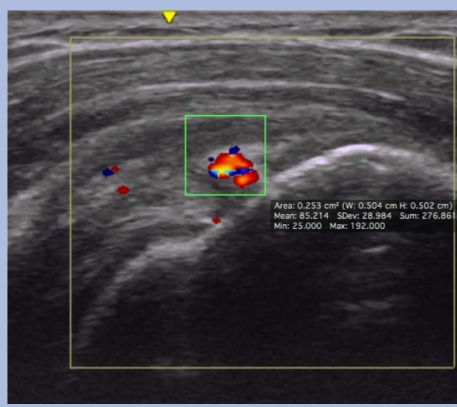

Grade 2 NV

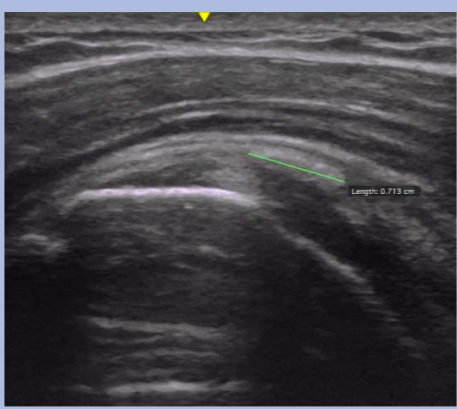

CA length measure

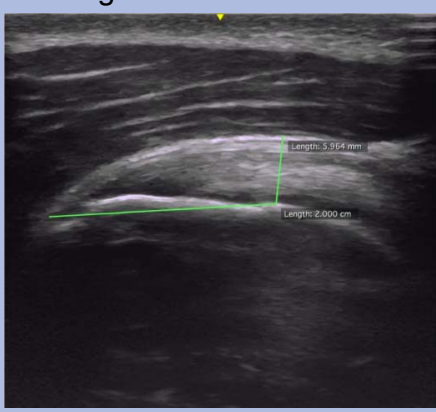

TT measure

CA, calcification; CC, caudal-cranial; FD, fibrillar disruption; GS, grey-scale; NV, neovascularity; PA, posterior-anterior; PD, Power Doppler; $\mathrm{ROI}$, Region of interest; TT, tendon thickness.

on 20 participants (10 cases and 10 controls), and the overall agreement of at least $80 \%$ on each parameter (present/not present for dichotomised variables, CA, NV, FD; no significant ( $p>0.05$ ) rater difference for continuous variables, TT, CA) was obtained before the actual reliability study.

\section{Study phase 3 (actual reliability study)}

\section{Participants}

General inclusion criteria were: $18-65$ years old; the ability to understand spoken and written Danish; no prior shoulder surgery/dislocation; no sensory or motor deficits in the neck/arm; no suspected competing 
Table 2 Grading scales with definitions for FD and NV

\begin{tabular}{lll}
\hline Grade & FD & NV \\
\hline 0 & Normal & Normal (no signal) \\
1 & Mild (involving under 25\% of the height of the tendon) & Mild (single small signal in the ROI) \\
2 & Moderate (involving $25-50 \%$ of the height of the tendon) & Moderate (Doppler activity in $<25 \%$ of the ROI) \\
3 & Severe (involving more than $50 \%$ of the height of the tendon) & Severe (Doppler activity in 25-50\% of the ROI) \\
4 & Partial rupture (disruption of the fibres in the full thickness of & Extreme (Doppler activity in more than $50 \%$ of \\
& the tendon) & the ROI) \\
\hline FD, fibrillar disruption; NV, neovascularity; ROI, region of interest. &
\end{tabular}

diagnoses (rheumatoid arthritis, cancer, neurological disorders, fibromyalgia, psychiatric illness).

Inclusion criteria for cases were: clinical diagnosis of RC tendinopathy with current shoulder symptoms lasting for at least 3 months prior to inclusion; pain located in the proximal lateral aspect of the upper arm (C5 dermatome) aggravated by shoulder abduction; positive 'full can test' and/or 'Jobe's test', and/or pain at 'resisted external rotation test'; and positive 'Hawkins-Kennedy test' and/or 'Neer's test'; and US verification of at least one of the following characteristics: FD, NV, CA (the involved side), or side difference (increased/decreased) TT of the supraspinatus tendon. $^{21}$

Exclusion criteria for cases were pain (during rest) rated above $40 \mathrm{~mm}$ (visual analogue pain scale, range $0-100 \mathrm{~mm}$ ); bilateral shoulder pain; $<90^{\circ}$ of active elevation of the arm; full thickness rupture in the supraspinatus tendon (verified by US); CA above $5 \mathrm{~mm}$ in the vertical distance (X-ray); corticosteroid injection within the latest 6 weeks; humerus fracture (X-ray); diagnoses of glenohumeral osteoarthritis; frozen shoulder; clinically suspected labrum lesion; symptomatic osteoarthritis in the acromioclavicular joint; or symptoms from the cervical spine. ${ }^{21}$

Inclusion criteria for controls were no shoulder discomfort within the latest 3 months and negative clinical shoulder tests.

Cases were consecutively recruited from specialised shoulder units at three hospitals in Denmark as part of a randomised controlled trial. ${ }^{21}$ Controls were recruited by advertisement among staff from The Department of Sports Science and Clinical Biomechanics, University of Southern Denmark, and the Rehabilitation Department, Lillebaelt hospital-Vejle hospital.

Informed consent was obtained from participants before inclusion.

\section{STATISTICS}

Linear weighted Cohen's $\kappa$ (LWk) was used to calculate inter-rater reliability with $95 \%$ CIs for the ordinal variables (FD, number of CA and NV). First, a linear weighing (LWk V.1) was applied, corresponding to the formula: $1-|\mathrm{i}-\mathrm{j}| /(\mathrm{k}-1)$, where $\mathrm{i}$ and $\mathrm{j}$ are the number of rows and columns, and $\mathrm{k}$ is the maximum number of possible ratings. ${ }^{22}$ Second, the same weighing was used
(LWk V.2), but with the restriction that disagreement between grades 0 and $>0$ was weighted as 0 , to account for the ability to differentiate between healthy and non-healthy.

The $\kappa$ was interpreted as $\leq 0.00=$ poor; 0.01 to $0.20=$ slight; 0.21 to $0.40=$ fair; 0.41 to $0.60=$ moderate; 0.61 to $0.80=$ substantial and 0.81 to $1.00=$ almost perfect. $^{23}$

For the continuous variables (TT, total length of CA), intraclass correlation (ICC; 3.1) was calculated as a measure of reliability. ICC was interpreted as $<0.40=$ poor, 0.40 to $0.75=$ fair to good and $>0.75=$ excellent reliability. ${ }^{24}$ Bland-Altman plots with $95 \%$ limits of agreement (LOA) were calculated as a measure of absolute agreement for TT (right and left) and total length of CA, and between-rater difference was tested by a paired t-test. Funnel effects and systematic bias were assessed visually and from Pearson's correlation coefficient (r). SEM was calculated as $\operatorname{SEM}_{(\text {Agreement) }}{ }^{25}$ to extrapolate results to the general population of potential raters, and minimal detectable change (MDC) was calculated at individual ( $\left.\mathrm{MDC}_{\text {Individual }}\right)$ and group $\left(\mathrm{MDC}_{\text {group }}\right)$ levels. ${ }^{26}$ Unpaired t-test was calculated for defining a potential cut-point of TT between cases and controls.

For the study phase, a sample size of 40 participants was applied, as previously recommended for reliability studies. $^{13}$

Data were analysed in Stata/IC V.14 (2015, Statacorp, College Station, Texas, USA). p Values $<0.05$ were considered significant.

\section{RESULTS}

There were no differences in demographics between cases and controls, except for pain and discomfort, as expected, due to the study design (table 3 ).

Total agreement ranged from $83 \%$ to $99 \%$, LWk V.1 for $\mathrm{FD}, \mathrm{NV}$ and CA ranged from 0.60 to 0.96 , and $\kappa$ with constraints (LWk V.2) varied from 0.51 to 0.98 , representing reliability of 'moderate-almost perfect' (table 4).

For total length of CA and TT, ICC ranged from 0.85 to 0.90 (excellent), with $\mathrm{SEM}_{\text {(Agreement) }}$ ranging from 0.63 to $2.94 \mathrm{~mm}, \mathrm{MDC}_{\text {(group) }}$ from 0.28 to $1.29 \mathrm{~mm}$ and $\mathrm{MDC}_{\text {(individual) }}$ from 1.75 to $8.15 \mathrm{~mm}$ (table 5).

No systematic rater differences were found in measured TT and total length of CA (table 5). Bland-Altman 
Table 3 Demographics (study phase; $n=40$ ))

\begin{tabular}{|c|c|c|c|}
\hline & Cases $(n=24)$ & Controls $(n=16)$ & p Value \\
\hline Sex (woman/men) & $10 / 14$ & $10 / 6$ & 0.20 \\
\hline Mean age (years) (SD) & $47.0(9.3)$ & $39.8(15.4)$ & 0.13 \\
\hline Height $(\mathrm{cm})(\mathrm{SD})$ & $176.2(10.75)$ & $171.9(7.8)$ & 0.18 \\
\hline Weight (kg) (SD) & $79.7(18.1)$ & $71.6(19.3)$ & 0.10 \\
\hline BMI & $25.4(3.6)$ & $24.1(5.7)$ & 0.12 \\
\hline Dominant arm right & $21 / 24$ & $14 / 16$ & 0.30 \\
\hline Duration of pain (months) (SD) & $24.3(34.9)$ & $0(0)$ & $<0.01$ \\
\hline VAS rest $(0-100)(S D)$ & $6.5(7.4)$ & $0(0)$ & $<0.01$ \\
\hline VAS activity (0-100) (SD) & $36.8(16.4)$ & $0(0)$ & $<0.01$ \\
\hline VAS sleep (0-100) (SD) & $30.0(23.6)$ & $0(0)$ & $<0.01$ \\
\hline VAS maximum (0-100) (SD) & $70.5(14.1)$ & $0(0)$ & $<0.01$ \\
\hline DASH (0-100) (SD) & $23.6(11.1)$ & $1.0(2.29)$ & $<0.01$ \\
\hline
\end{tabular}

plots showed no funnel effects, but a small interaction between difference and increased mean was found for TT in the left shoulder $(\mathrm{r}=0.35, \mathrm{p}=0.03$; figure 2$)$. In general, LOA showed a larger variation for CA than for TT (table 5 and figure 2).

No significant difference was found between cases and controls in TT.

\section{DISCUSSION}

The inter-rater reliability study showed moderate to perfect reliability for grading $\mathrm{FD}, \mathrm{NV}$ and number of $\mathrm{CAs}$, using standardised procedures. Inter-rater reliability for measuring the total length of CA and TT was excellent, and MDC indicated small detectable changes for group level, especially in TT.

\section{FD and hypoechoic areas}

Despite merging hypoechoic areas and FD into one scale, reliability was still only moderate (LWk of 0.60 and 0.51 ). This was, however, in line with previous studies of tendinopathy, where agreement on subtle changes ('mild abnormality' and 'normal') was considered especially difficult, presumably due to difficulties in differing structural changes and anisotropy. ${ }^{4} 681011$ Grading FD may be more easily interpreted with in vivo US examinations, as the examiner is more flexible when evaluating presence of anisotropy.

\section{Neovascularisation}

The current reliability of NV was almost perfect. The reason for the high reliability in the current study may be the grading of $\mathrm{NV}$ in relation to a predetermined ROI (fixed box of $5 \times 5 \mathrm{~mm}$ placed over the area with most NV), as opposed to grading NV relative to the TT or the tendon in general as previously in tendinopathy of the elbow. ${ }^{4} 16$ The current modification was performed to increase standardisation, as well as to account for between and within variations in TT, of interest in intervention studies.

Other studies have found prevalence of NV in $30-65 \%$ of symptomatic shoulders with only $25 \%$ of asymptomatic shoulders. ${ }^{27} 28$ This study found prevalence of NV in $38 \%$ of the cases and $0 \%$ in the control group. This large variation in prevalence across previous studies may be due to different populations, PD settings, measurement methods and the position of the participant arm during US image capturing. This study placed the hand at the sacrum, to maximally stretch the supraspinatus tendon, which may have increased the risk of overlooking $\mathrm{NV}$ due to restricted flow in the neovessels. Different study designs across studies make it difficult to compare prevalence and establish normative levels for use in clinical practice.

Table 4 Inter-rater reliability of grading presence of FD, NV and number of CAs (study phase; $n=40$ )

\begin{tabular}{|c|c|c|c|}
\hline Ordinal scale & $\begin{array}{l}\text { Total agreement } \\
\text { (LWк V.1) (\%) }\end{array}$ & $\begin{array}{l}\text { LWk } \\
(\mathrm{V} .1) \\
(95 \% \mathrm{Cl})\end{array}$ & $\begin{array}{l}\text { LWк (V.2) } \\
\text { (95\% Cl) }\end{array}$ \\
\hline FD & 83.3 & 0.60 (0.40 to 0.79$)$ & 0.51 (0.30 to 0.72$)$ \\
\hline $\mathrm{CA}$ & 93.8 & 0.72 (0.59 to 0.85$)$ & 0.75 (0.56 to 0.89$)$ \\
\hline NV & 99.2 & $0.96(0.85$ to 1.0$)$ & $0.98(0.93$ to 1.0$)$ \\
\hline
\end{tabular}

LWא (V.1): no cut-point applied in the weights schedule.

LW $(V .2)$ : cut-point applied in weights when raters $A$ and $B$ disagree between grades 0 and $>0$.

$\mathrm{CA}$, calcification; FD, fibrillar disruption; LWא, linear weighted $\kappa ; ~ N V$, neovascularity. 


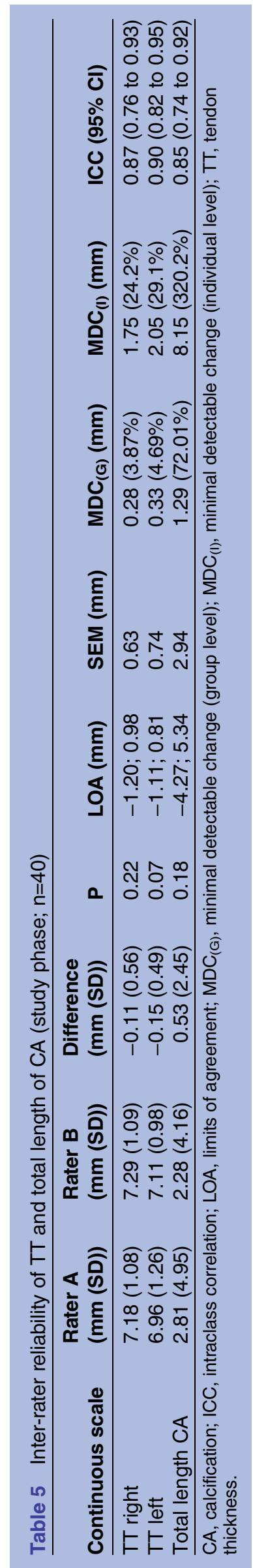

\section{Calcification}

The substantial $\kappa$ for detecting the total number of CA is in line with previous studies, ${ }^{4} 8$ but LOA, SEM and MDC showed considerable variation on the total length of CA. This variation may be due to US methodological problems, for example, that shadows underneath CA may falsely be interpreted as FD and/or normal tendon structure may appear hyperechoic, thus resembling CA, which may result in misclassifications. However, reliability of number of CA was high, indicating that measuring individual lengths of CA and/or few undetected/misclassified CA have influenced agreement of total length of CA. One outlier seen in the Bland-Altman plots (figure 2) indicates that raters $\mathrm{A}$ and $\mathrm{B}$ disagreed on at least one larger structural change, which, owing to the generally small size and low prevalence of CA, has influenced the variation considerably.

\section{Tendon thickness}

Excellent reliability, and MDC of $\leq 0.33 \mathrm{~mm}$, indicates that the variable is sensitive for detecting changes, in line with a previous study using the same method for measuring $\mathrm{TT}^{18}{ }^{18}$ This means that it may be a clinically relevant measurement for assessment of changes in tendon properties, such as increased/decreased oedema. Some studies have found significant differences in TT between symptomatic and non-symptomatic participants, ${ }^{29} \quad 30$ which are in contrast with the current study and a recent study. ${ }^{18}$ The reason for the discrepancy across studies may be due to the use of different methods for measuring TT, small sample sizes, different inclusion criteria or, as in this study, the inclusion of more active controls (recruited among health personnel) with potentially thicker tendons than the average population.

One limitation of the study is the transferability to clinical setting, as this study used captured images and strictly standardised procedures, which are rarely used in clinical settings. In vivo, raters would be more flexible when evaluating presence of anisotropy in the interpretation of potential $\mathrm{FD}$; also, they would be able to perform repeated image capturing and measurements when $\mathrm{CA}$ or $\mathrm{NV}$ were suspected to be present. Use of a standardised protocol for reliability studies ${ }^{13}$ may be a weakness, since reliability of the current US method and procedures may have been deceptively high compared with a clinical setting. However, if the standardised method has poor reliability in a standardised setting, reliability is also assumed to be poor and the method less relevant for use in a clinical setting. The raters measured and graded the captured images and movies on different DICOM viewers. Whether this has influenced the reliability is unknown. However, since reliability is found to be high on most variables, it is considered to not be important and to mimic clinical practice.

The strengths of this study are the design, incorporating a stepwise and standardised procedure in order to minimise bias and increase reliability. ${ }^{13}$ The present standardisation of US image and movie capturing, 

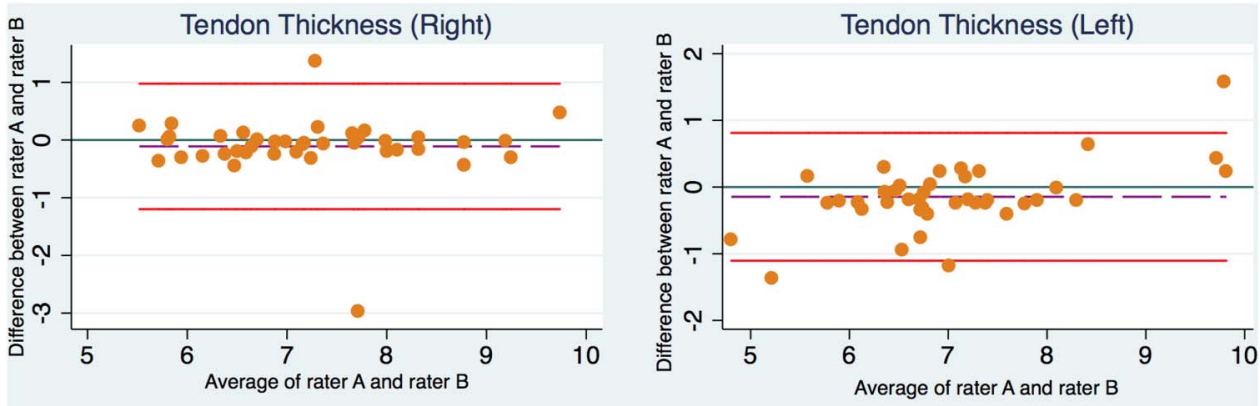

Figure 2 Bland-Altman plots with 95\% LOA for TT (right and left) and total length of CAs. CA, calcifications; LOA, limits of agreement; TT, tendon thickness.

measuring and grading structural changes are anticipated to increase reliability and sensitivity of the method. Despite one of the raters having relatively few years of US experience, reliability was still high and satisfactory, indicating that the protocol can even be followed by other than very US-experienced clinicians. By using captured images and movie sequences, it was ensured that both raters had equal underlying bases for interpretation of the reliability study.

Further, the use of weighing with restrictions when calculating $\mathrm{\kappa}$ was considered important, due to the importance of being able to differ between cases and controls.

\section{CONCLUSION}

Inter-rater reliability was moderate to almost perfect when a standardised procedure was applied for measuring structural changes on captured US images and movie sequences of relevance for patients with supraspinatus tendinopathy. Future studies should test intra-rater and inter-rater reliability of the method in vivo for use in clinical practice, in addition to validation against a gold standard, such as MRI.

\section{Author affiliations}

${ }^{1}$ Department of Sports Science and Clinical Biomechanics, University of Southern Denmark, Odense, Denmark

${ }^{2}$ Department of Rehabilitation, Hospital Lillebaelt-Vejle Hospital, Vejle, Denmark

${ }^{3}$ Department of Radiology, Musculoskeletal section, Odense University Hospital, Odense, Denmark

${ }^{4}$ Health Sciences Research Centre, University College Lillebaelt, Odense, Denmark

${ }^{5}$ Shoulder Unit, Orthopaedic Department, Hospital Lillebaelt, Vejle Hospital, Vejle, Denmark
${ }^{6}$ Department of Health Sciences, Institute of Occupational Therapy, Physiotherapy and Radiography, Bergen University College, Bergen, Norway

Contributors KGI, BJ-K, HE and CML conceived and designed the study protocol. KGI and BJ-K procured the project funding. KGI, BJ-K, JV and JH developed and standardised the ultrasound procedure and defined the grading scale. JV and JH secured access and coordinated screening procedures at the shoulder units. KGI was the project coordinator and performed the inclusion and US image capturing. $\mathrm{KGI}$ and $\mathrm{JH}$ were raters. $\mathrm{KGI}$ and BJ-K planned and coordinated the statistical analyses. KGI performed the statistical analyses. $\mathrm{KGI}$ drafted the manuscript, and BJ-K, JH, JV, HE and CML contributed to the manuscript. All authors read and approved the final manuscript. KGI is the guarantor.

Funding Region of Southern Denmark's Research fund, The Danish Rheumatism Association and the Ryholts Foundation funded the trial.

Competing interests None declared.

Patient consent Obtained.

Ethics approval All procedures performed in studies involving human participants were in accordance with the ethical standards of the institutional and/or national research committee and the 1964 Helsinki declaration and its later amendments or comparable ethical standards. The Regional Scientific Ethics Committee of Southern Denmark has approved the trial (project ID: S-20130071).

Provenance and peer review Not commissioned; externally peer reviewed.

Data sharing statement No additional data are available.

Open Access This is an Open Access article distributed in accordance with the Creative Commons Attribution Non Commercial (CC BY-NC 4.0) license, which permits others to distribute, remix, adapt, build upon this work noncommercially, and license their derivative works on different terms, provided the original work is properly cited and the use is non-commercial. See: http:// creativecommons.org/licenses/by-nc/4.0/

\section{REFERENCES}

1. Cook JL, Purdam CR. Is tendon pathology a continuum? A pathology model to explain the clinical presentation of load-induced tendinopathy. Br J Sports Med 2009;43:409-16. 
2. Lewis JS. Rotator cuff tendinopathy: a model for the continuum of pathology and related management. $\mathrm{Br} J$ Sports Med 2010;44:918-23.

3. Hegedus EJ, Goode A, Campbell S, et al. Physical examination tests of the shoulder: a systematic review with meta-analysis of individual tests. Br J Sports Med 2008;42:80-92; discussion 92.

4. Poltawski L, Ali S, Jayaram V, et al. Reliability of sonographic assessment of tendinopathy in tennis elbow. Skeletal Radiol 2012;41:83-9.

5. Ottenheijm RP, Jansen MJ, Staal JB, et al. Accuracy of diagnostic ultrasound in patients with suspected subacromial disorders: a systematic review and meta-analysis. Arch Phys Med Rehabil 2010;91:1616-25.

6. Naredo E, Möller I, Moragues C, et al. Interobserver reliability in musculoskeletal ultrasonography: results from a "Teach the Teachers" rheumatologist course. Ann Rheum Dis 2006;65:14-19.

7. Ottenheijm RP, van't Klooster IG, Starmans LM, et al. Ultrasound-diagnosed disorders in shoulder patients in daily general practice: a retrospective observational study. BMC Fam Pract 2014;15:115.

8. O'Connor PJ, Rankine J, Gibbon WW, et al. Interobserver variation in sonography of the painful shoulder. J Clin Ultrasound 2005;33:53-6.

9. Thoomes-de Graaf M, Scholten-Peeters GG, Duijn E, et al Inter-professional agreement of ultrasound-based diagnoses in patients with shoulder pain between physical therapists and radiologists in the Netherlands. Man Ther 2014;19:478-83.

10. O'Connor PJ, Grainger AJ, Morgan SR, et al. Ultrasound assessment of tendons in asymptomatic volunteers: a study of reproducibility. Eur Radiol 2004;14:1968-73.

11. Sunding K, Fahlstrom M, Werner S, et al. Evaluation of Achilles and patellar tendinopathy with greyscale ultrasound and colour Doppler: using a four-grade scale. Knee Surg Sports Traumatol Arthrosc 2014 [Epub ahead of print]

12. Weinreb $\mathrm{JH}$, Sheth $\mathrm{C}$, Apostolakos J, et al. Tendon structure disease, and imaging. Muscles Ligaments Tendons J 2014;4:66-73.

13. Patijn JV, Beek JV, Blomberg S, et al. Reproducibility and validity studies of diagnostic procedures in manual/musculoskeletal medicine. In: Patijn J, ed. International Federation for manual/ musculoskeletal medicine. 2004:3-35.

14. Ohberg L, Alfredson $\mathrm{H}$. Ultrasound guided sclerosis of neovessels in painful chronic Achilles tendinosis: pilot study of a new treatment. Br J Sports Med 2002;36:173-5; discussion 176-7.

15. Venu KM, Howlett DC, Garikipati R, et al. Evaluation of the symptomatic supraspinatus tendon-a comparison of ultrasound and arthroscopy. Radiography 2002;8:235-40.

16. Krogh TP, Fredberg U, Stengaard-Pedersen K, et al. Treatment of lateral epicondylitis with platelet-rich plasma, glucocorticoid, or saline: a randomized, double-blind, placebo-controlled trial. Am J Sports Med 2013;41:625-35.

17. Ottenheijm RP, Joore MA, Walenkamp GH, et al. The Maastricht Ultrasound Shoulder pain trial (MUST): ultrasound imaging as a diagnostic triage tool to improve management of patients with non-chronic shoulder pain in primary care. BMC Musculoskelet Disord 2011;12:154.

18. Hougs Kjaer B. Intra-rater and inter-rater reliability of standardized ultrasound protocol for assessing subacromial structures (Submitted after revision). Physiother Theory Pract 2017. In press.

19. Ingwersen KG, Hjarbaek J, Eshoej $\mathrm{H}$, et al. Sonographic assessment of supraspinatus tendinopathy as a future diagnostic and effect measure - a reliability study of the assessment methode. $X X V$ Congress of the International Society of Biomechanics; Glasgow, UK: ISB, 2015.

20. Martinoli C. Musculoskeletal ultrasound: technical guidelines. Insights Imaging 2010;1:99-141.

21. Ingwersen KG, Christensen R, Sørensen L, et al. Progressive high-load strength training compared with general low-load exercises in patients with rotator cuff tendinopathy: study protocol for a randomised controlled trial. Trials 2015;16:27.

22. Sim J, Wright CC. The kappa statistic in reliability studies: use, interpretation, and sample size requirements. Phys Ther 2005;85:257-68.

23. Landis JR, Koch GG. The measurement of observer agreement for categorical data. Biometrics 1977;33:159-74.

24. Fleiss JL. Reliability of measurement. The design and analysis of clinical experiments. Hoboken, NJ: John Wiley \& Sons, Inc., 1999.

25. de Vet HC, Terwee CB, Knol DL, et al. When to use agreement versus reliability measures. J Clin Epidemiol 2006;59:1033-9.

26. de Vet HCW TC, Mokkink LB, Knol DLL. Measurement in medicine -a practical guide. New York, USA: Cambridge University Press, New York, 2011

27. Kardouni JR, Seitz AL, Walsworth MK, et al. Neovascularization prevalence in the supraspinatus of patients with rotator cuff tendinopathy. Clin J Sport Med 2013;23:444-9.

28. Lewis JS, Raza SA, Pilcher J, et al. The prevalence of neovascularity in patients clinically diagnosed with rotator cuff tendinopathy. BMC Musculoskelet Disord 2009;10:163.

29. Arend CF, Arend AA, da Silva TR. Diagnostic value of tendon thickness and structure in the sonographic diagnosis of supraspinatus tendinopathy: room for a two-step approach. Eur $J$ Radiol 2014;83:975-9.

30. Michener LA, Subasi Yesilyaprak SS, Seitz AL, et al. Supraspinatus tendon and subacromial space parameters measured on ultrasonographic imaging in subacromial impingement syndrome. Knee Surg Sports Traumatol Arthrosc 2015;23:363-9. 